\title{
Theoretical Modeling of a photodetector based on ballistic Carbone nanotube with VHDL-AMS
}

\author{
M. Troudi ${ }^{2}$, A. Mahmoudi ${ }^{1}$, N. Sghaier ${ }^{1,2, a}$ A.Soltani ${ }^{3}$ \\ ${ }^{1}$ Laboratoire de Microélectronique et Instrumentation (LR13ES12), Faculté des Sciences de \\ Monastir, Avenue de l'environnement, Université de Monastir, 5019 Monastir, Tunisia. \\ ${ }^{2}$ Equipe composants électroniques (UR/99/13-22), Institut Préparatoire aux Etudes d'Ingénieurs de \\ Nabeul (IPEIN), Université de Carthage, 8000 Merazka, Nabeul, Tunisia. \\ ${ }^{3}$ Institut d'Electronique, Microélectronique et Nanoélectronique, Unité Mixte de Recherche CNRS \\ 8520 -Université des Sciences et Technologies de Lille Avenue Poincaré - BP 60069 - 59652 \\ Villeneuve d'Ascq cedex
}

E-mail address: nabil.sghaier @ipein.run.tn

Keywords: Theoretical Modeling; photodetector based; ballistic Carbone; VHDL-AMS

\begin{abstract}
In this paper we present a new VHDL-AMS model of carbone nanotube field effect transistor for photo-detection application: (photo-CNTFET). Contrary to classical photodetectors, the photo-CNTFET has the potential to work on a wide range of optical frequencies and high quantum efficiency and can be used as a highly sensitive and rapid response photodetector. Based on its excellent conductivity and very low capacitance, Carbon nanotubes provide highly mobile electrons and low noise in the system. The simulation results obtained in the present paper has shown its relevance as precise and fast tool to investigate the effects of photoexcitation on $\mathrm{Ids}_{\mathrm{d}} \mathrm{V} \mathrm{ds}$ characteristics of the photo-CNTFET. We have present results obtained after variation of power illumination and light beam wavelength.
\end{abstract}

\section{INTRODUCTION}

Carbon nanotubes (CNTs), discovered in 1991, have been a subject of intensive research for a wide range of applications [1,2]. A very large literature has been published on the properties of CNTs. These tubes may be found in two types of structures: multi- walled nanotubes (MWCNTs) and single-walled nanotubes (SWCNTs). The Single-walled carbon nanotubes (SWCNTs) are nearly ideal one-dimensional (1D) materials [3, 4]. The carrier confinement and reduced dimensionality give them unique electrical properties that have been, in particular used as channels of field-effect transistors [2-6]. The major operation, Structure and operation of CNTFET is the same as traditional MOSFET. Because of the one-dimensional material of the CNT, electrons are limited to the narrow nanotube, the mobility goes up greatly on account of ballistic transport, as compared with the traditional bulk MOSFET [7]. We will consider the MOSFET-like CNTFET with ballistic transport as our device of interest.

So far, Among the wide range of application of CNTs, semiconducting Carbon Nanotubes show interesting optoelectronic properties In particular from near ultraviolet (UV) to infrared (IR) [8]. Their ability to generate a photocurrent in a wide wavelength range has been demonstrated by several authors [9-12] [13-19]. In this paper we have developed a compact model of a new photo-detector based on carbon nanotube field effect transistor photo-CNTFET characterized by a ballistic conduction.

This model is defined and developed in VHDL-AMS language. It represents an important amelioration of the absorption of light in the CNT structures which is our matter of interest. The novelty of this photo-CNTFET resides on its highly sensitivity to the radiation, from UV to IR wavelength region. We show that measurements of photocurrent demonstrate the ability of our device to detect visible light radiation. We also demonstrate that the simulation results obtained using this compact model, presents important amelioration of photocurrent at hight power illumination and higher wavelength. 


\section{PHOTO-CNTFET DETECTOR}

For photodetection application, Photo carbon nanotube field effect transistor (photoCNTFET) appears as a good candidate according to its high charge sensitivity and excellent conductivity.

The device structure presented in this paper is consisting of a single walled carbon nanotube incorporated as the channel of a field-effect transistor (FET). Our compact model of the photoCNTFET is developed based on the one of CNTFET [20]. Under illumination, the self consistent voltage Vsc of the photo-CNTFET is directly affected by the electron-hole pairs which are generated within the carbon nanotube molecule.

The study of the charge balance in the presence of the light explains the mechanism of the modulation of the potential channel VSC due to the optical excitation.

$$
\begin{aligned}
& \mathrm{Q}(\quad)=\mathrm{C}_{\mathrm{G}} \mathrm{V}_{\mathrm{G}}+\mathrm{C}_{\mathrm{S}} \mathrm{V}_{\mathrm{S}}+\mathrm{C}_{\mathrm{D}} \mathrm{V}_{\mathrm{D}}+\mathrm{C}_{\mathrm{J}} \mathrm{V}_{\mathrm{ph}} \\
& C_{\Sigma}=C_{G}+C_{S}+C_{D}+C_{J} \\
& V_{S C}(\lambda)=\square \frac{Q(\lambda)+q N_{S}\left(V_{S C}(\lambda)\right)+q N_{D}\left(V_{S C}(\lambda)\right)+q N_{0}}{C_{\Sigma}}
\end{aligned}
$$

with $\mathrm{VG}_{\mathrm{G}}, \mathrm{VD}, \mathrm{VS}$ and $\mathrm{Vph}$ are respectively the potentials of gate, drain, source and Laser (excitation source). The four products terms respectively represent the charges issue from the gate, the drain, the source and the optical source. Here $C \Sigma$ is the total terminal capacitance.

$$
C_{o x}=\frac{2 \pi \varepsilon_{1} \varepsilon_{0}}{\cosh \square\left(\frac{2 t_{o x}}{d}\right)}
$$

where'd' is the diameter of the CNT, $t S u b$ is the thickness of the $\mathrm{SiO}_{2}$ layer on the substrate, $t_{O x}$ is the thickness of the gate insulator and, are the relative permitivities of the gate and the substrate respectively. $\mathrm{CD}$ and $\mathrm{CS}$ present the electrostatic parasitic capacitances of drain and source respectively. We have assumed that $\mathrm{CS}=\mathrm{CD}$ due to the symmetry of the device.

$Q S$ and $Q D$ are the non-equilibrium mobile charge densities. They can be modeled as nonlinear circuit capacitances, which are dependent on the self-consistent voltage VSC.

$$
\begin{aligned}
& Q_{S}\left(V_{S C}\right)=q\left(N_{S}\left(V_{S C}\right) \square \frac{1}{2} N_{0}\right) \\
& Q_{D}\left(V_{S C}\right)=q\left(N_{D}\left(V_{S C}\right) \square \frac{1}{2} N_{0}\right)
\end{aligned}
$$

Where $N S$ (respectively $N D$ ) is the density of positive (negative) velocity states filled by the source (drain), and $N_{0}$ is the equilibrium electron density. 
The other elements shown in the equivalent circuit of the photo-CNTFET (Fig.1) RG, RD, and RS are access resistances to the gate, drain, and source, respectively.

refers to the self consistent voltage Vsc. $C_{O X}$ is the planer gate capacitor. The nodes $G, D$, and $\mathrm{S}$ refer to the intrinsic CNTFET device.

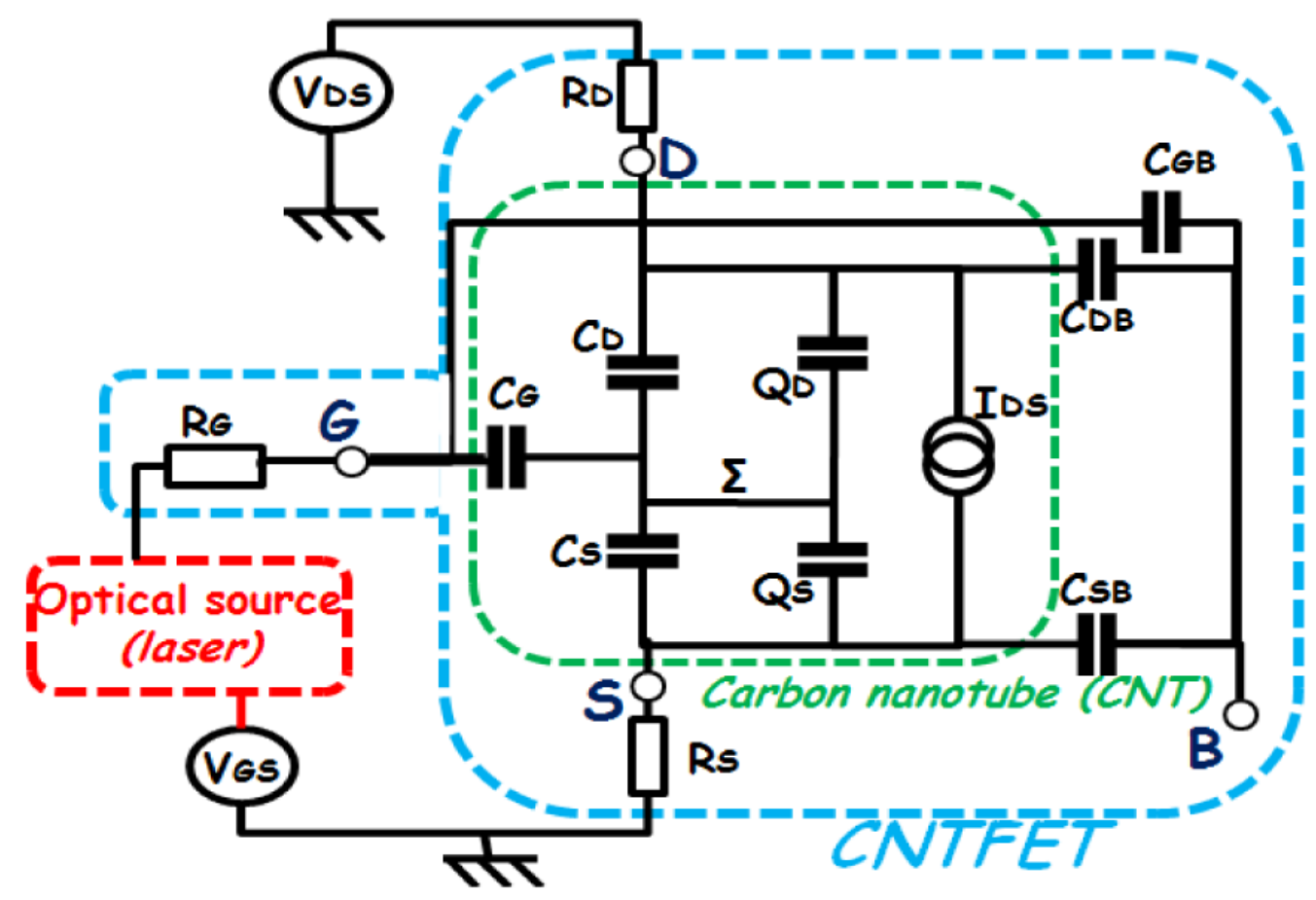

Fig. 1. Schematic representation of the proposed photo-CNTFET detector

The working principle of the proposed photo-CNTFET is based on ballistic transport of charges between the CNT and the electrodes via the gate and can be gated by input optical intensity.

The photo-CNTFET shows a significant increase of the drain current. The photo generated carriers are separated by an applied electric field between the source and the drain contacts. Under important power illumination the light excitation will dominate the functionality of the photo-CNTFET. Thus the optical characteristic values of the light power will replace the gate effect and will behave as a photonic gate. The photocurrent $I p h$ is directly proportional to the incident optical power Popt and the quantum efficiency of electron generating and the photon energy which is linked directly to the laser wavelength:

$$
I_{p h}=P_{o p} t \eta_{e x t} \frac{q}{h v}=P_{o p t} \eta_{e x t} \frac{\lambda(\mu m)}{1.24}
$$

Iph results from the photo generated electron-hole pairs.

This current source was used to excite the second exciton state of the semiconducting nanotube ((E22) transition). When this state is excited with an energy $E=h \omega>\mathrm{E}_{22}$ and absorbed by the CNT, an energetic e-h pair at the band edge will diffuse within the channel or drift in an applied electric field [21].

\section{RESULTS AND DISCUSSIONS}

VHDL-AMS is a derivative of the hardware description language VHDL (Very High Speed Integrated Circuit (VHSIC) Hardware Description Language). It includes analog and mixedsignal extensions $(A M S)$ in order to define the behavior of analog and mixed-signal systems [22]. 
To investigate the device properties as radiation detector, we have used the photo-CNTFET to measure the curves characteristics $\mathrm{Ids}_{\mathrm{ds}}-\mathrm{V}_{\mathrm{gs}}$ in the dark and under laser illumination to show the effect of the optical gate.

Fig. 2 shows the curves of photocurrent measured when the device is illuminated with a continuous laser beam at $532 \mathrm{~nm}$ for various light power intensities swept from 0.1 to $1 \mathrm{~mW}$ with a step of $0.1 \mathrm{~mW}$.

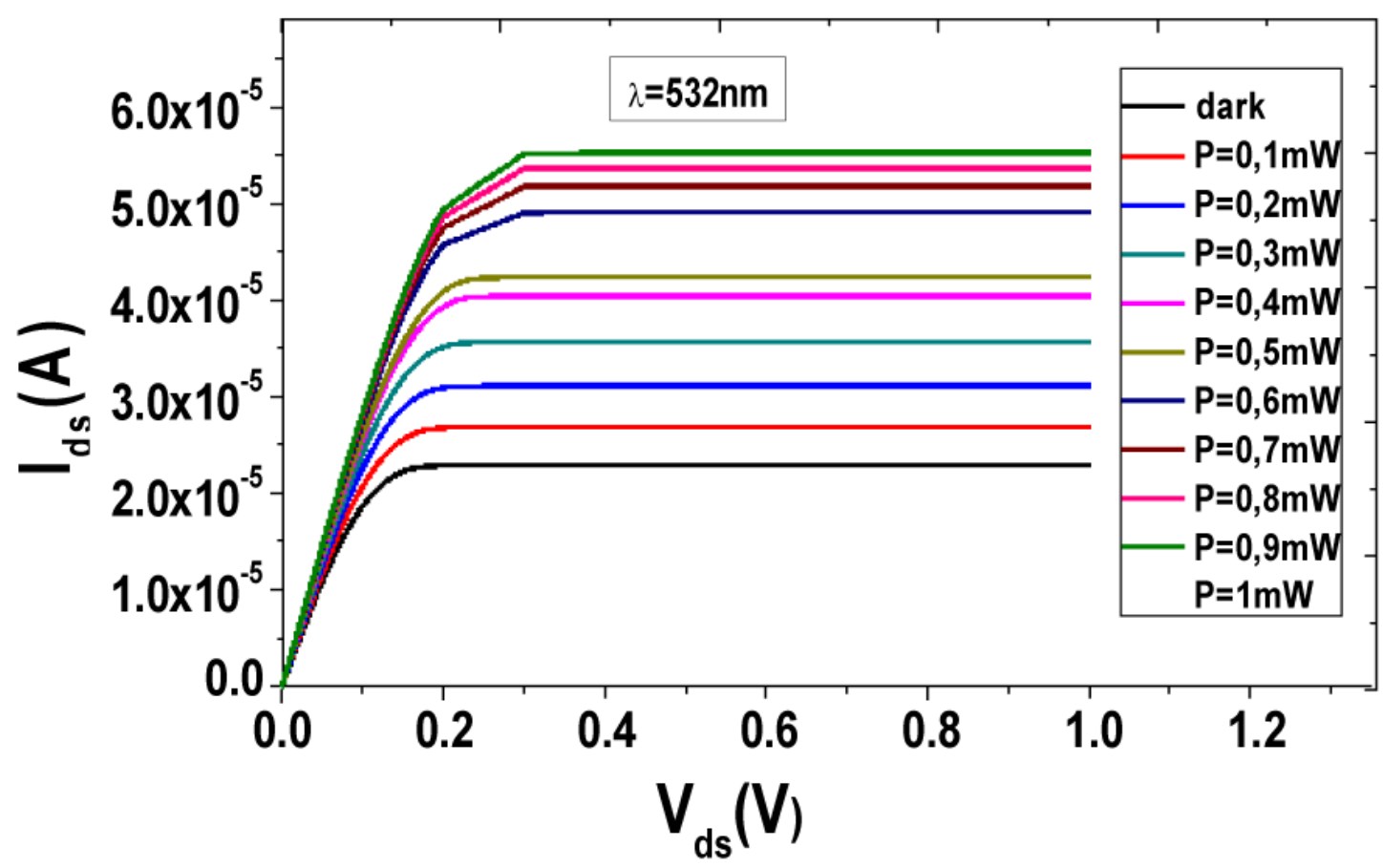

Fig. 2. $\mathrm{Ids}-\mathrm{V}$ ds curves at $\mathrm{L}=532 \mathrm{~nm}$ for various laser light intensities

In order to reduce photo generated electrons-holes recombination and to collect and detect the maximum of photo carriers, we apply a moderate drain voltage on the order of 0.6 $\mathrm{V}$. This draining voltage limits carrier injection from the contacts into the channel and allows the photo-generated carriers to leave the channel and be collected. It can be seen that the generated signal is typical of a phototransistor: the drain current increases as the drain voltage until a voltage value from which the photo-current becomes constant. This constant current explains that all the photo-generated carriers are collected at the electrodes. In the region of saturation our device operates as an ideal photo detector in which the output signal depends only on the intensity of radiation.

Under excitation, a large part of the incident light will be absorbed by the CNT. Indeed this absorbed light will generate carriers and giving rise to the photocurrent.

Fig. 3.a shows a direct proportionality between the current at saturation versus the laser light power. As shown on this figure, the same linear behavior has been found at all the laser light wavelengths. This result is in agreement with the relation between the photocurrent $I \mathrm{ph}$ and the incident optical power $P_{\text {opt }}$ (9). For higher light intensities the photo-current is important, thus the charges have acquired significant energy while increasing light power enabling them to overcome the barriers at the interface between the CNT and contacts. In addition, the transport in the CNT is ballistic. It allows photo-generated carriers to cross the carbon nanotube with minimum of interaction between them and reduce the probability of a recombination process. Fig. 3.b shows the evolution of photo-current with the laser light wavelengths at different optical power. It can be seen that in all cases, the saturation current value as well as the incident power increases with the incident wavelength of the laser beam in a linear relation. Thus, the 
photocurrent exhibits a linear response with the light wavelength and with bias voltage varying from to $0.5 \mathrm{~V}$ to $0.75 \mathrm{~V}$. For higher wavelengths, we will have a large electric field in the middle of photo-CNTFET device which will separate the carriers and limit the charge recombination, making the photocurrent maximized and the detector noiseless in this operating condition.
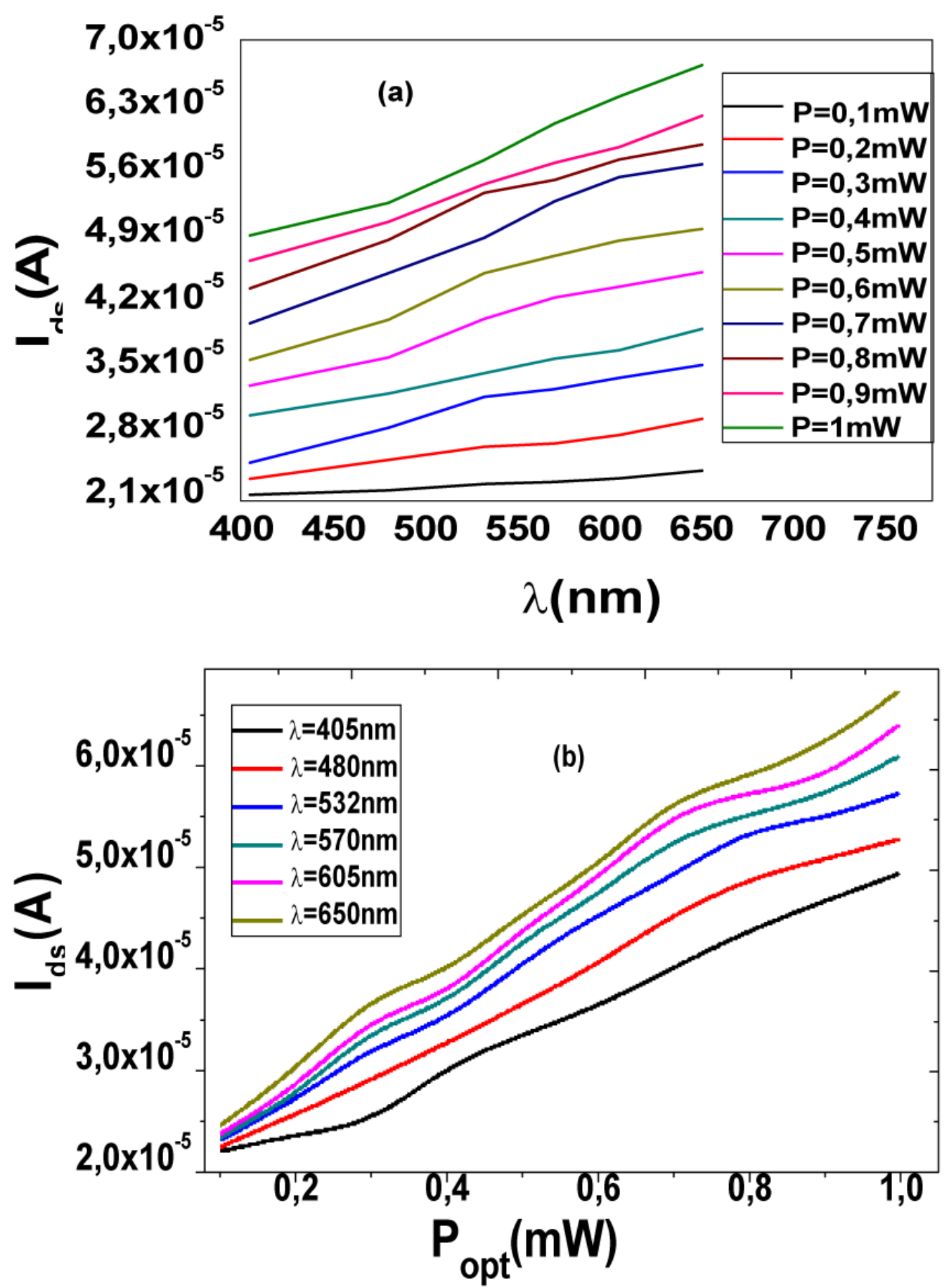

Fig. 3: (a) The effect of incident optical power showing the increscent of photo-generated current and linearity plot at various wave lengths.(b) Effect of laser beam wavelength on the photcurrent .

\section{CONCLUSION}

The present photo-CNTFET is extremely promising in a future use in high energy physics applications. It allows large photosensitive areas with the desired geometry. The performance of this detector depends on the carbon nanostructure morphology and 
ultimately on their electronic properties. The photocurrent is strictly proportional to the light power intensity almost up to $1 \mathrm{~mW}$ in the wavelength range $405-650 \mathrm{~nm}$. Only a low drain voltage varying between 0.5 to $0.75 \mathrm{~V}$ is needed to drive a photocurrent of about $6.9 \mu \mathrm{A}$ for a $1 \mathrm{~mW}$ source power at $650 \mathrm{~nm}$ light beam wavelength.

Our photo-CNTFET can be immediately produced on industrially scale, and used at room temperature as high light sensor. We note that our device does not require signal amplification.

\section{References}

[1] S. Iijima, "Helical microtubules of graphitic carbon”, (1991), Nature, No 56 ,pp354.

[2] S. Iijima and T. Ichihashi, "Single-shell carbon nanotubes of $1 \mathrm{~nm}$ diameter",(1993), Nature, No 603, pp 363.

[3] S. Reich, C. Thomsen, J. Maultzsch, "Carbon Nanotubes: Basic Concepts and Physical Properties", Wiley-VCH, New York, 2003. [4] R. Saito, G. Dresselhaus, M.S. Dresselhaus, "Physical Properties of Carbon Nanotubes", Imperial College Press, 2003.

[5] F. Sharifi, A. Momeni, K. Navi, "CNFET based basic gates and a novel full-adder cell", International Journal of VLSI design \& Communication Systems (VLSICS), (2010) Vol. 3, No. 3, pp 11-19.

[6] Ghorbani, M. Sarkhosh, E. Fayyazi, P. Keshavarzian, (2012) "A novel full adder cell based on carbon nanotube field effect transistors", International Journal of VLSI design \& Communication Systems (VLSICS), Vol. 3, No. 3, pp 33-42.

[7] Avouris P, Appenzeller J, Martel R, Wind SJ. "Carbon nanotube electronics". Proceedings of IEEE. 2004; Vol 91,No11, pp1772-1784.

[8] A. Ambrosio, C. Aramo. "Carbon Nanotubes-Based Radiation Detectors, Carbon Nanotubes Applications on Electron Devices", ISBN:978-953-307-496-2, InTech(2011).

[9] P. Castrucci et al., "Large photocurrent generation in multiwall carbon nanotubes", Appl. Phys(2006). Lett. pp89, No 253107.

[10] P. Castrucci et al., "Light harvesting with multiwall carbon nanotube/silicon heterojunctions", Nanotechnology (2011), pp 22, No 115701.

[11] M. Tzolov et al., "Carbon nanotube-silicon heterojunction arrays and infrared photocurrent responses", J. Phys. Chem. C (2007), pp 111, No 5800.

[12] P.L. Ong, W.B. Euler and I.A. Levitsky, "Carbon nanotube-Si diode as a detector of midinfrared illumination”, Appl. Phys. Lett. (2010), pp 96 No,033106.

[13] A. Ambrosio et al., "Sensing pulsed light by means of multi-walled carbon nanotubes", Mat. Sci. Semicon. Proc. (2008), pp 11, No 187.

[14] M. Passacantando et al., "Photoconductivity in defective carbon nanotube sheets under ultraviolet-visible-near infrared radiation”, Appl. Phys. Lett. (2008),pp 93, No 051911.

[15] B.K. Sarker, M. Arif, P. Stokes and S.I. Khondaker, "Diffusion mediated photoconduction in multiwalled carbon nanotube films", J. Appl. Phys. (2009),pp 106,No 074307.

[16] S. Lin, Y.B. Kim, F. Lombardi, (2009) "A novel CNTFET-based ternary logic gate design", $52^{\text {nd }}$ IEEE International Midwest Symposium on Circuits and Systems, pp 435-438.

[17] M.E. Itkis et al., "Bolometric infrared photoresponse of suspended single-walled carbon nanotube film”, Science(2006), pp 312, No 5772. 
[18] S.X. Lu and B. Panchapakesan, "Photoconductivity in single wall carbon nanotube sheets",Nanotechnology(2006), pp17, No 1843.

[19] I.A. Levitsky and W.B. Euler, "Photoconductivity of single-wall carbon nanotubes under continuous-wave near-infrared illumination", Appl. Phys. Lett. (2003), pp83 No.1857.

[20] T. J. Kazmierski, D. Zhou, B. M. Al-Hashimi, and P. Ashburn, "Numerically Efficient Modeling of CNT Transistors With Ballistic and Nonballistic Effects for Circuit Simulation," IEEE Transactio tel-00592479.

[21] M. Freitag, Y. Martin, J. A. Misewich,,| R. Martel, and Ph. Avouris, "Photoconductivity of Single Carbon Nanotubes”, NANO LETTERS 2003 Vol. 3, No. 81067-1071

[22]www.mentor.com 